\title{
Gut chemosensing mechanisms
}

\author{
Arianna Psichas, Frank Reimann, and Fiona M. Gribble \\ Cambridge Institute for Medical Research, Addenbrooke's Hospital, Cambridge, United Kingdom.
}

\begin{abstract}
The enteroendocrine system is the primary sensor of ingested nutrients and is responsible for secreting an array of gut hormones, which modulate multiple physiological responses including gastrointestinal motility and secretion, glucose homeostasis, and appetite. This Review provides an up-to-date synopsis of the molecular mechanisms underlying enteroendocrine nutrient sensing and highlights our current understanding of the neuro-hormonal regulation of gut hormone secretion, including the interaction between the enteroendocrine system and the enteric nervous system. It is hoped that a deeper understanding of how these systems collectively regulate postprandial physiology will further facilitate the development of novel therapeutic strategies.
\end{abstract}

\section{The enteroendocrine system}

The enteroendocrine system is the primary sensor of ingested food and is responsible for secreting an array of gut hormones, which act in concert to modulate multiple physiological responses including gastrointestinal motility and secretion, glucose homeostasis, and appetite (Figure 1). An impressive number of gut hormones has been implicated in these processes including cholecystokinin (CCK), glucagon-like peptide 1 (GLP1), glucosedependent insulinotropic peptide (GIP), peptide YY (PYY), somatostatin, ghrelin, and serotonin (5-hydroxytryptamine [5-HT]). While the discovery of these hormones dates back to the 1970s and 1980s, they have returned to the spotlight following the association of some with the dramatic weight loss and diabetes remission observed as a consequence of bariatric surgery (1).

The enteroendocrine system forms the largest endocrine organ in the body and plays a vital role in the regulation of postprandial physiology, yet its intricate workings remain shrouded in mystery. This can be partly attributed to the elusive nature of enteroendocrine cell (EEC) populations, which collectively account for just $1 \%$ of intestinal epithelial cells. However, the field has made significant advances in recent years owing largely to the engineering of transgenic mice in which the expression of fluorescent proteins is driven by enteroendocrine hormone promoters $(2-5)$. The use of this technology has shed light on several novel aspects of EEC biology and has challenged traditional concepts in the field. One such dogma was the notion that EECs belong to distinct cell types such as the GLP1- and PYY-secreting L cells, CCK-secreting I cells, and GIP-secreting K cells. Surprisingly, following characterization of proglucagon-Venus, GIP-Venus (6), and CCK-eGFP (7) reporter mice, it is now clear that individual EECs, particularly in the small intestine, are capable of expressing a much broader range of gut hormone precursors, opening up the exciting possibility of therapeutically manipulating not only levels of endogenous gut hormone secretion but also EEC type and number (reviewed in ref. 8). Despite the rejection of the "one cell type,

Conflict of interest: The authors have declared that no conflict of interest exists. Reference information: / Clin Invest. 2015;125(3):908-917. doi:10.1172/JCI76309. one hormone" hypothesis, gut hormones exhibit characteristic patterns of expression along the gastrointestinal tract. While some interspecies differences do exist, GIP is typically found at higher levels in the duodenum, CCK in the duodenum and jejunum, and GLP1 in the jejunum, ileum, and colon. PYY, on the other hand, is predominantly expressed in the distal ileum and colon $(9,10)$.

An additional rich source of EECs is the stomach. Examples of gastric hormone-producing cells include chief cells and $\mathrm{P}$ cells, which produce leptin (11-13); D cells and G cells, which release somatostatin and gastrin, respectively $(14,15)$; and A cells (X-like), which secrete ghrelin, an appetite-stimulating hormone with additional roles in energy storage and glucose homeostasis (16-18). The actions and importance of intestinal leptin, which is secreted in response to food intake (11), are currently an active area of research (19). Until recently ghrelin, and possibly motilin, were thought to be the only orexigenic gut hormones. However, a recent study identified insulin-like 5 (INSL5) as an additional orexigenic gut peptide, produced by colonic L cells in response to caloric restriction. INSL5 is thought to increase food intake by acting on the relaxin family peptide receptor (RXFP4) (20).

EECs are found scattered along the gastrointestinal epithelium, and most exhibit the characteristic "open-type" structure, with a narrow apical surface facing the gut lumen and a broader basolateral surface from which dense-core secretory vesicles are exocytosed (21). A notable exception is the "closed-type" ghrelinsecreting cell that does not come into contact with the gastric lumen (16). Basolaterally released gut hormones activate enteric, vagal, and spinal sensory neurons via their respective receptors or enter the circulation to act in an endocrine fashion. Once in the subepithelial space, gut hormones can also signal in a paracrine manner to neighboring epithelial cells. A well-characterized example is the activation of PYY Y1 receptors located on enterocytes, which leads to the inhibition of cAMP-dependent $\mathrm{Cl}^{-}$secretion (22). EECs often possess dendrite-like processes, which are thought to serve paracrine and sensory functions (23). Indeed, the recent employment of a PYY-GFP transgenic mouse line in conjunction with high-resolution confocal microscopy revealed a novel feature of EEC morphology: a prominent basal cytoplasmic process, referred to as a "neuropod," possessing axon-like traits 


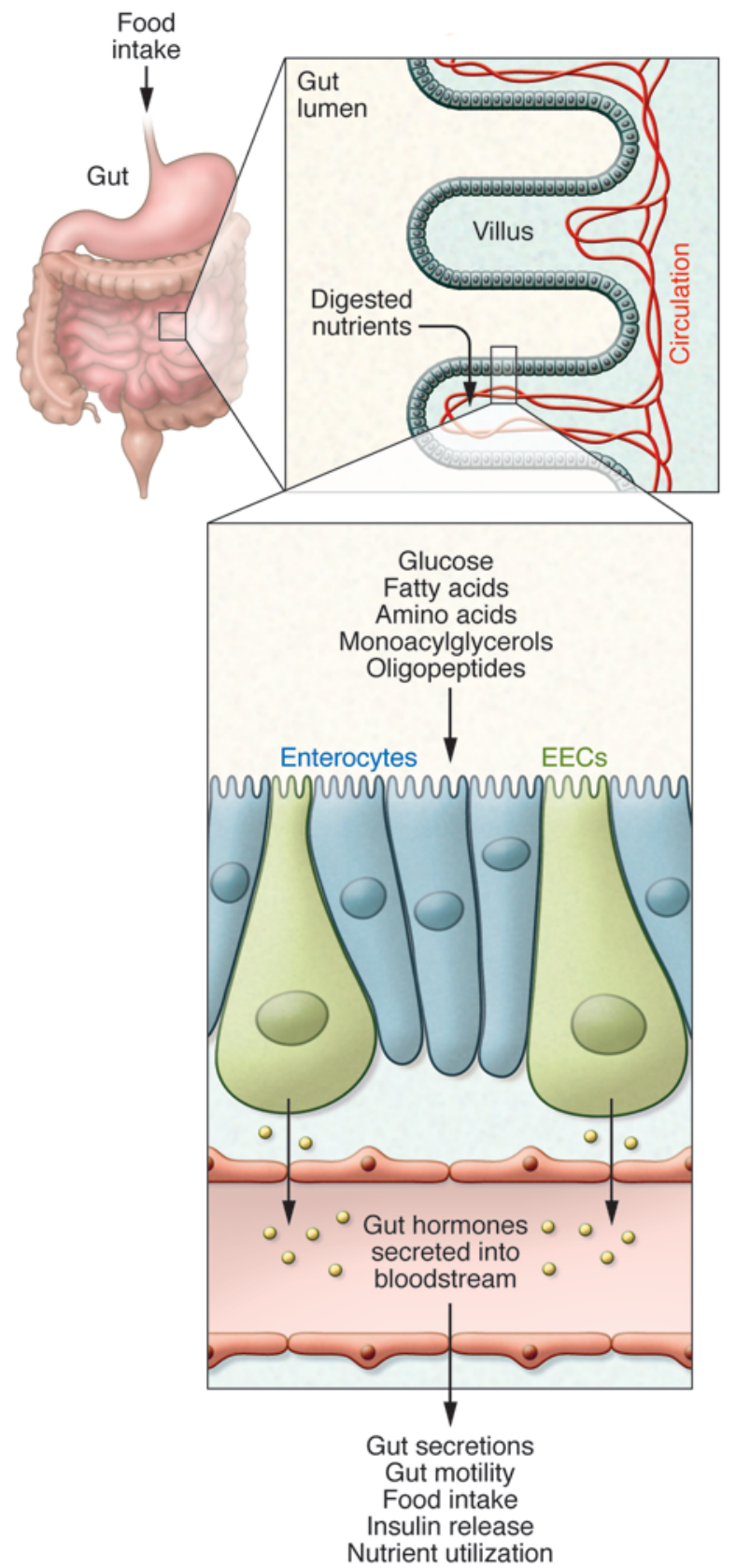

including a putative physical interaction with glial fibrillary acidic protein-expressing enteric glia $(2,24)$. While the functional role of the neuropod structure remains to be determined, the possibility of discovering novel cell-to-cell interactions that modulate EEC secretion or action opens exciting avenues for future investigation.

EECs are directly activated by their exposure to nutrients arriving in the gut lumen, and the consequent secretion of gut hormones serves as a physiological signal to alert the body to the arrival of absorbed nutrients. Detection of the luminal contents has been attributed to the presence on EECs of sensory transporters and receptors, as well as to the utilization of nutrients by EECs as energy substrates. Surrounding enterocytes may also release active metabolites or deliver stimuli to the sub-epithelial space following their absorption across the epithelium. Indeed, for most nutrient-sensing receptors it remains unclear whether
Figure 1. Nutrient sensing by the enteroendocrine system. Ingested food is digested into its nutrient components in the lumen of the small intestine. The small intestinal epithelium is arranged in villi containing, among other cell types, absorptive enterocytes and EECs. The presence of nutrients in the gut lumen stimulates EECs and triggers the secretion of gut hormones, which orchestrate the body's postprandial response. Gut hormones modulate multiple physiological processes including gastrointestinal secretion and motility, insulin release, and satiety.

they are predominantly located on the apical or basolateral surface of EECs. This Review focuses on the mechanisms underpinning nutrient and non-nutrient chemosensing, with a focus on the release of the incretins GLP1 and GIP, which act on pancreatic $\beta$ cells to enhance glucose-stimulated insulin secretion.

\section{Molecular mechanisms underlying gut chemosensing}

Nutrient sensing. Investigation into enteroendocrine nutrientsensing pathways has gained momentum in recent years, particularly in vitro. A large number of GPCRs have been identified as EEC nutrient sensors, and a growing body of evidence suggests that primary L cells are electrically active, generating nutrient responses characterized by action potential firing and voltagegated calcium influx $(5,25)$.

Carbohydrates. Carbohydrates are predominantly sensed in the form of glucose. In humans, glucose is a potent stimulator of GLP1 and GIP secretion, though it is less consistent at triggering a rise in plasma CCK (26-28). A number of mechanisms for glucosestimulated incretin release have been proposed (Figure 2A), and there has been a lively debate regarding the contribution made by the luminal presence of glucose and its uptake/absorption or metabolism. The mechanism for which there is the most support is $\mathrm{Na}^{+}$-coupled glucose uptake via the sodium/glucose cotransporter member 1 (SGLT1). An important role for absorption and the requirement for luminal $\mathrm{Na}^{+}$ions in glucose-stimulated gut hormone secretion were determined in earlier studies, which demonstrated that GLP1 and GIP secretion was mediated by both metabolizable and non-metabolizable substrates of SGLT1 (29-31). Recent evidence suggests that small transporter-triggered currents stimulate membrane depolarization and voltage-gated $\mathrm{Ca}^{2+}$ entry $(5,32)$. Glucose-stimulated GLP1 and GIP secretion in vitro is prevented by pharmacological inhibition of SGLT1 (33), and Sglt1-deficient mice exhibit impaired GLP1 and GIP secretion immediately following oral glucose administration (34). At later time points after glucose gavage, plasma GLP1 was paradoxically increased in Sglt1 knockout mice (35), perhaps because reduced glucose absorption in the upper small intestine results in increased nutrient delivery to the L cell-rich distal gut, where an SGLT1-independent pathway may play a more important role.

$\mathrm{L}$ and $\mathrm{K}$ cells also express glucokinase and ATP-sensitive potassium $\left(\mathrm{K}_{\mathrm{ATP}}\right)$ channel subunits, and a potential role for glucose metabolism was suggested by in vitro experiments using the model cell line GLUTag (33). However, metabolism-dependent pathways are not thought to be responsible for peak GLP1 and GIP concentrations measured early after an oral glucose load, and incretin levels in humans were not affected by treatment with $\mathrm{K}_{\text {ATP }}$ channel blockers (36). Changes in plasma glucose have little effect 
A

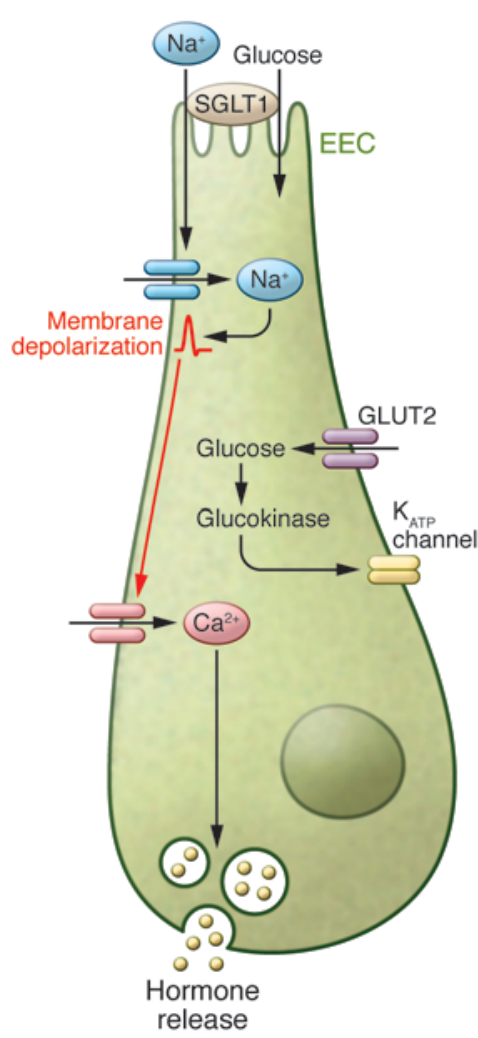

B

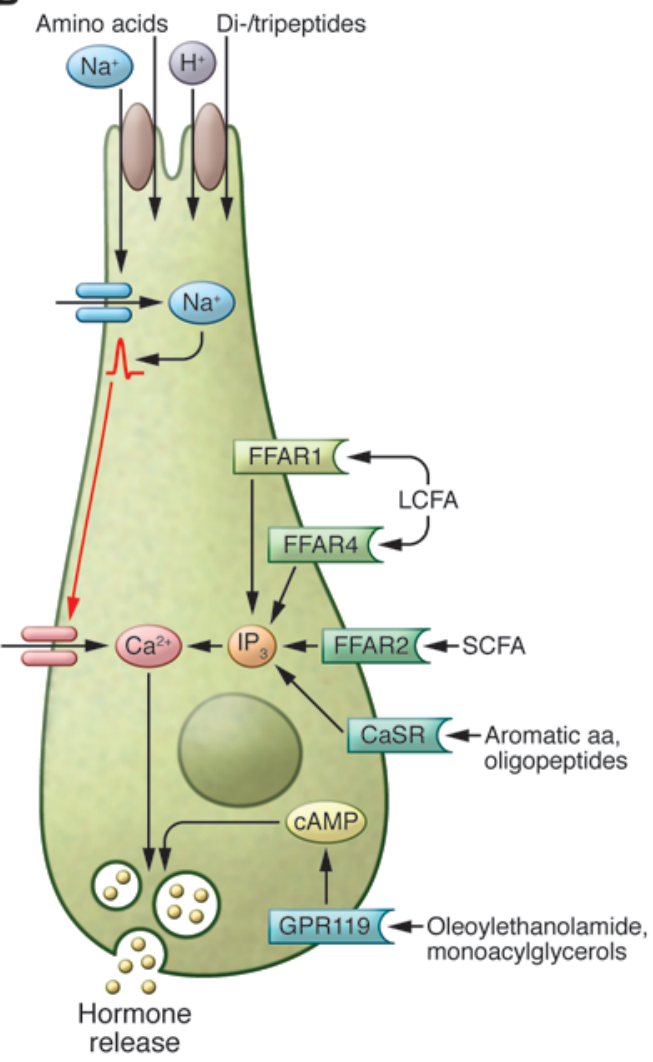

Figure 2. Glucose, fat, and amino acid sensing by EECs. (A) Glucose sensing by EECs involves a number of mechanisms. A critical component of glucose sensing in the gut is $\mathrm{Na}^{+}$-coupled glucose uptake by SGLT1, which generates small currents that trigger depolarization and voltagegated $\mathrm{Ca}^{2+}$ entry. Glucose metabolism, involving glucokinase and the closure of ATP-sensitive $\left(\mathrm{K}_{\mathrm{ATP}}\right)$ channels, and basolateral/plasma glucose concentration may also play a role in glucosestimulated gut hormone release. (B) There are several pathways by which fatty acids and amino acids are sensed by EECs. Fatty acids activate nutrient-sensing GPCRs, which include FFAR1 (GPR40) and FFAR4 (GPR120) for MCFAs and LCFAs and FFAR2 (GPR43) for SCFAs, leading to an increase in intracellular $\mathrm{Ca}^{2+}$. Activation of GPR119 by oleoylethanolamide and monoacyl glycerols stimulates gut hormone secretion via an increase in intracellular cAMP. Similarly, amino acids and oligopeptides can also activate GPCRs such as the CaSR. In addition, electrogenic uptake of certain amino acids and dipeptides and tripeptides can also trigger membrane depolarization and gut hormone release. on plasma incretin levels (33), although small GLP1 responses triggered by oral fructose might be attributable to metabolism (37), as apical fructose uptake is mediated by a non-electrogenic transporter GLUT5. Overall, our current understanding of the sensory importance of EEC metabolism remains limited.

Activation of the sweet taste receptor, a G-protein-coupled heterodimer (T1R2/T1R3) that senses glucose and artificial sweeteners, has also been proposed to stimulate gut hormone secretion. This hypothesis was driven by studies that detected sweet taste receptor machinery, such as the G-protein $\alpha$-gustducin, in the gut, some of which also demonstrated co-localization with GLP1 and GIP (38-40). Whereas $\alpha$-gustducin (38) and T1r3 knockout mice (41) exhibited impaired glucose-stimulated GLP1 secretion, glucose homeostasis in T1r2-deficient mice was normal (41), and there is currently insufficient evidence to support direct sensing of sweet taste by K or L cells (5). In addition, several studies have concluded that the ingestion of glucose, but not artificial sweeteners, increases plasma GLP1 and GIP levels in rats (42) and humans (43), findings which at present argue against an important role for sweet taste detection in gut hormone secretion.

Proteins. Dietary protein is a potent stimulus of CCK release and also stimulates secretion of GLP1 and GIP, although with some inconsistencies between different reports. High-protein meals are associated with markedly increased plasma PYY levels and enhanced satiety (44). However, the mechanisms underlying protein-induced gut hormone secretion are relatively poorly understood, and the optimal size of the products of protein digestion for maximal stimulation of gut hormone release has yet to be established. It is clear that amino acids as well as pep- tides of varying sizes are capable of activating EECs, implying the existence of multiple sensory pathways (Figure 2B). Several amino acids have been demonstrated to stimulate GLP1 secretion in vitro $(25,45)$. The L-glutamine-triggered GLP1 pathway is perhaps one of the best characterized and is thought to involve $\mathrm{L}$ cell membrane depolarization through electrogenic $\mathrm{Na}^{+}$-dependent transport, as well as elevation of $\operatorname{cAMP}(25,45)$. In humans, ingestion of L-glutamine raises plasma GLP1 levels in healthy, obese, and diabetic subjects (46). Amino acid-stimulated gut hormone secretion has been associated with activation of the primarily $\mathrm{G}_{\mathrm{q}}$-coupled calcium-sensing receptor (CaSR) (47). The CaSR is best known for its role in sensing extracellular $\mathrm{Ca}^{2+}$, but is also expressed in EECs, where it is gaining a reputation as an amino acid sensor (47-49). Additional candidates for mediating GLP1 release in response to amino acids include the umami taste receptor dimer T1R1/T1R3 (50) and GPRC6A (51).

The mechanisms involved in the sensing of larger protein digestion products, such as di- and tripeptides and oligopeptides, are less clear. Meat hydrolysate has been demonstrated to trigger GLP1 release from vascularly perfused rat small intestine, STC-1 cells (a small intestine enteroendocrine murine cell line) (52), and NCI-H716 cells (derived from human colorectal adenocarcinoma) (53), as well as CCK release from I cells (54). The sensing of these larger protein digestion products has been linked to the activation of MAPKs $(52,53)$ and the activity of the brush border $\mathrm{H}^{+}$-coupled transporter of dipeptides and tripeptides, peptide transporter 1 (PEPT1) $(54,55)$. Both PEPT1- and CaSR-dependent pathways were recently implicated in the sensing of peptones and dipeptides and tripeptides by primary L cells (56). Another receptor, lyso- 
phosphatidic acid receptor 5 (LPAR5, also known as GPR92/93), was reported to mediate peptone-stimulated CCK release in Lpar5-overexpressing STC-1 cells (57), although its expression is not enriched in native I cells (49) or L cells (56). As GLP1 secretion was found not to be impaired in primary colonic cultures from Lpar5 knockout mice (56), further work is required to elucidate what role, if any, LPAR5 plays in intestinal chemosensing.

Lipids. The ingestion of fat, which is subsequently hydrolyzed by lipases in the small intestine, is a strong stimulus of gut hormone secretion (58-60). Several GPCRs have been identified as sensors of the products of fat digestion (Figure 2B). In EECs, these include GPR120 (FFAR4) (61), GPR119 $(62,63)$, and FFAR1 (GPR40) (64). GPR120 and FFAR1 respond to unsaturated longchain fatty acids (LCFAs) and medium-chain fatty acids (MCFAs), and both are thought to couple to $\mathrm{G}_{\mathrm{q}}$-proteins and thus activate phospholipase $\mathrm{C}$, leading to $\mathrm{IP}_{3}$-mediated $\mathrm{Ca}^{2+}$ release from intracellular stores $(61,65)$. GPR120 and FFAR1 expression is largely restricted to EECs in the intestinal epithelium, including $\mathrm{I}, \mathrm{L}$, and $\mathrm{K}$ cells (3-5), and a role in gut hormone secretion for these receptors has been demonstrated in murine and human colonic cultures and in vivo $(61,66)$. Mice lacking FFAR1 exhibited impaired release of GIP and GLP1 on a high-fat diet (64), and LCFA-induced CCK release from ffar1-deficient I cells was compromised (3). It is currently unclear which of these two receptors plays a more important role in intestinal lipid sensing. Interestingly, recent work revealed that ghrelin cells express GPR120, which is linked to inhibition of secretion from primary gastric mucosal cultures (48).

Gpr119 mRNA is enriched in $\mathrm{K}$ and $\mathrm{L}$ cells $(4,5,62)$ and is thought to be activated by oleoylethanolamide and 2-monoacylglycerols, which are derived from triglycerides (67). GPR119 is a $\mathrm{G}_{\mathrm{s}}$-coupled GPCR-activating adenylyl cyclase that increases intracellular cAMP levels $(62,68,69)$. cAMP is a well-documented trigger for gut hormone secretion and gene transcription via pathways coupled to the recruitment of protein kinase A and EPAC2 (70-72). Pharmacological activation of GPR119 using synthetic smallmolecule agonists increased both plasma GLP1 and GIP concentrations in mice (62). Oral administration of 2-oleoylglycerol increased plasma GLP1 and GIP levels in humans (67), however, a GPR119 agonist failed to stimulate GLP1 and PYY secretion from human colonic cultures (66), and the efficacy of GPR119 agonists in clinical trials has been underwhelming (73).

Alternative lipid-sensing pathways have also been implicated in EECs. These include contributions from fatty acid transporter, member 4 (74), activation of atypical PKC $\zeta$ (75), and indirect stimulation by chylomicrons and other lipoproteins released by neighboring enterocytes. In support of the latter hypothesis, administration of the hydrophobic surfactant and inhibitor of intestinal chylomicron formation Pluronic L-81 (76) led to reduced lipidinduced GIP secretion in mice (77) and rats (78), and in I cells, the regulation of CCK release by fatty acids was lipoprotein dependent and was attributed to activation of immunoglobulin-like domain containing receptor 1 (79). Mice lacking monoacylglycerol acyltransferase 2 or diacylglycerol acyltransferase 1 (DGAT1), enzymes that sequentially resynthesize triglycerides to be incorporated into chylomicrons, also exhibited impaired GIP release in response to an oral triglyceride load. By contrast, enhanced GLP1 and PYY responses were observed in Dgat1-deficient mice (80), and following inhibition of microsomal triglyceride transfer protein in rats on a high-fat diet background (81). It thus now seems almost certain that lipid ingestion activates a multitude of sensory pathways in EECs, and further work will be required to dissect the relative physiological importance and translation potential of individual components.

\section{Sensing of non-nutrient stimuli}

EECs sense a variety of non-nutrient luminal and paracrine signals. These include inflammatory cytokines, enteral progesterone (82), and bile acids (BAs) as well as gut hormones and enteric neurotransmitters.

A number of studies identified IL-6 as a cytokine potentially involved in the modulation of glucose homeostasis $(83,84)$. Increased IL-6 levels observed during exercise coincided with increased concentrations of plasma $\operatorname{GLP} 1(85,86)$. Recent work has revealed that the IL-6 receptor is expressed in GLUTag cells and incubation with IL-6 increases both GLP1 production and secretion in these cells (87). Elevated IL-6 levels might, therefore, contribute to the increased GLP1 concentrations observed during exercise.

BAs play a critical role in the emulsification and digestion of lipids and are believed to act as non-nutrient stimuli of L cells. In humans, administration of BAs has been associated with increased plasma GLP1 levels and improved glucose homeostasis $(88,89)$. The increased plasma BAs and enhanced BA delivery to the distal gut observed following gastric bypass surgery may contribute to the elevated GLP1 and PYY levels associated with gut rearrangement procedures $(90,91)$. BAs are thought to stimulate GLP1 release via activation of the predominantly $G_{s}$-coupled receptor GPBAR1 (also known as TGR5), which is enriched in L cells, particularly in the distal intestine $(92,93)$.

Gut hormone secretion from EECs is also under the influence of signals from other EEC populations, such as somatostatin arising from intestinal D cells (14). In the stomach, somatostatin is an important regulator of gastrin secretion from neighboring $\mathrm{G}$ cells (15), whereas in the intestine it likely acts over greater distances as a paracrine and/or hormonal factor. Five somatostatin receptors (SSTRs) have been identified, all thought to be $\mathrm{G}_{\mathrm{i}}$-coupled, leading to inhibition of adenylyl cyclase and a reduction in intracellular cAMP levels. In GLUTag and primary L cells, somatostatin is believed to inhibit GLP1 and PYY release via SSTR5 (94). Ghrelin secretion is also inhibited by somatostatin, potentially involving SSTR1, -2, and -3 (48). The effects of other gut hormones on ghrelin release are varied (95-97). GIP, for example, has been reported as both an inhibitor and stimulator of ghrelin secretion. The recent identification in ghrelin cells of the GIP receptor, which is predominantly $G_{s}$ coupled, would suggest that any direct action of GIP on ghrelin cells is likely to be stimulatory (48) and that any inhibitory effects observed in situ might occur via indirect pathways.

\section{Gut microbiota, host defense, and EEC signaling}

The mammalian large intestine harbors more than 1,000 different species of bacteria, collectively known as the gut microbiota. In recent years gut microbiota have been increasingly recognized as a fundamental component of host metabolism, and shifts in bacterial populations have been associated with endocrine disorders such as obesity and type 2 diabetes (98-101). Gut microbiota 


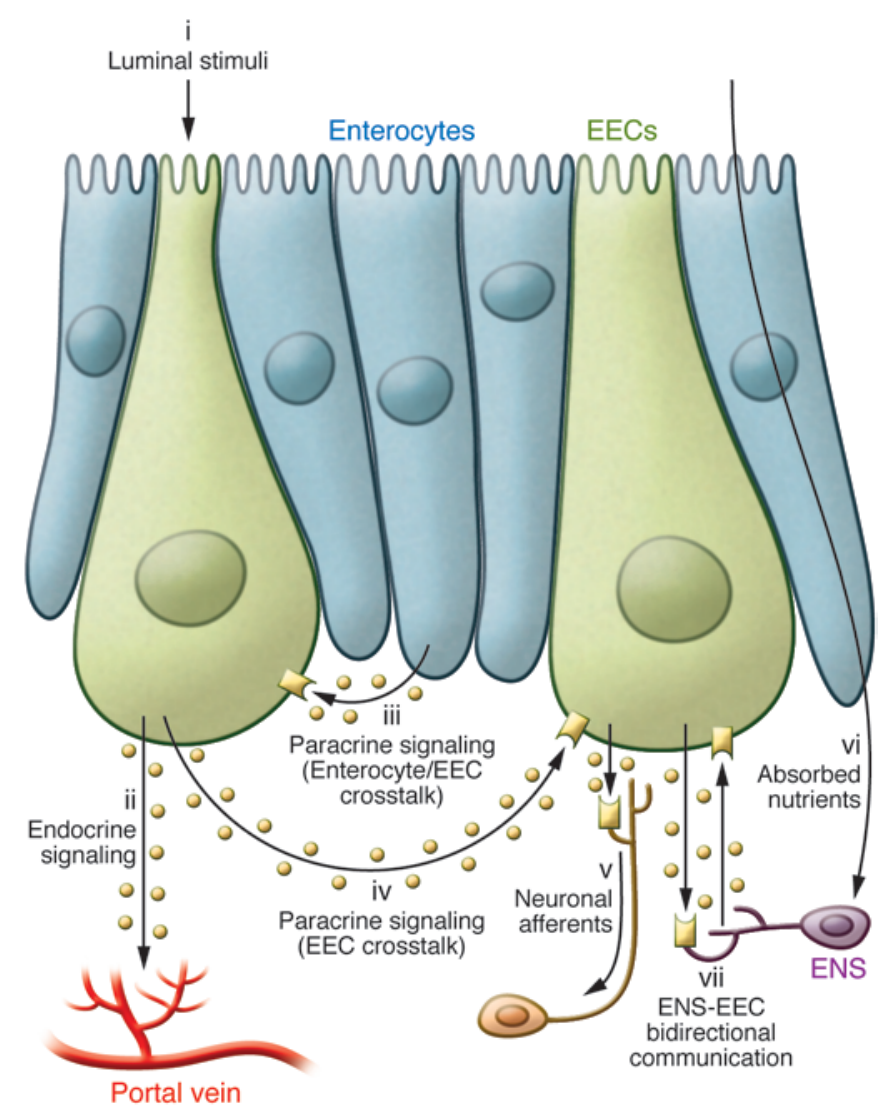

release a large variety of metabolites that potentially interact with EECs located in the distal intestinal epithelium.

Short-chain fatty acids (SCFAs), including acetate, propionate, and butyrate, are the major end products of microbial fermentation of non-digestible carbohydrates and have received considerable attention as key candidates for microbiota-host signaling. They have been identified as ligands for two GPCRs, free fatty acid receptor 2 (FFAR2, previously known as GPR43) and FFAR3 (previously known as GPR41) $(102,103)$, both of which are detectable in rat and human colonic mucosa (104-106) and in murine $L$ cells (106-108). FFAR2 couples to $G_{q}$ and $G_{i}$ pathways, whereas FFAR3 is presumed to couple exclusively to the pertussis toxin-sensitive $G_{i}$ pathway $(102,103)$. Both receptors have also been demonstrated to activate MAPK $(103,109)$, which has been associated with GLP1 release from EEC cell lines in response to a number of stimuli $(82,110)$. SCFA-dependent GLP1 secretion was significantly impaired in Ffar2 knockout mice, which also exhibited reduced circulating GLP1 concentrations, supporting a role for FFAR2 signaling in GLP1 secretion $(108,111)$.

Interestingly, Ffar2/3 mRNA appears also to be enriched in small intestinal I cells and gastric ghrelin cells $(48,112)$. In contrast to the effects on L cells, FFAR2 agonists inhibit ghrelin secretion via $\mathrm{G}_{\mathrm{i}}$-coupled mechanism. Engelsoft and colleagues hypothesized that this shift toward $G_{i}$ coupling may result from the high expression and enrichment of non-classical $G_{i}$ subunits in ghrelin cells (48).

In addition to SCFAs, gut bacteria contribute a vast array of heterogeneous metabolites including secondary BAs, phenols, choline metabolites, indole derivatives, vitamins, neurotransmitters and
Figure 3. Neuroendocrine crosstalk in the gut. The secretion of gut hormones from EECs is stimulated by the sensing of luminal factors (i), (e.g., nutrients, bile acids, microbial products) predominantly via apical GPRCs $\left(G_{q} / G_{s}\right)$ and transporters. Gut hormones secreted into the subepithelial space mediate their local and systemic effects via a number of signaling pathways. These include absorption into the circulation to act in the classical (ii) endocrine manner; (iii) paracrine signaling involving activation of surface receptors (e.g. NPY1R on enterocytes; SSTR5 on L cells; immunoglobulin-like domain containing receptor 1 [ILDR1] on I cells) on the basolateral surface of neighboring enterocytes and/or other EECs (iv); and activation of GPCRs (e.g. GLP1R, NPY2R, CCK1R) on vagal afferents (v) to relay information centrally, or on enteric neurons to modulate intestinal function. Enteric neurons are also capable of sensing certain absorbed nutrients directly (vi). For example, subsets of enteric neurons express the SCFA receptor FFAR3. In turn, the ENS fine-tunes the function of EECS (vii), as evident from their expression of a number of neuropeptide receptors (e.g., galanin receptor [GALR1], bombesin receptors [BB2R], vasoactive intestinal peptide receptor 1 [VPAC1R]).

neurotransmitter precursors, and bioactive lipids (113, 114). Indeed, microbiota-derived indole, produced by bacterial degradation of dietary tryptophan, has recently been shown to exert dual effects on GLP1 release - a stimulatory action mediated by voltage-gated $\mathrm{K}^{+}$channel blockade and an inhibitory effect caused by suppression of mitochondrial metabolism (115). However, our understanding of how the complex "soup" of bacterial metabolites interacts with host cells, including EECs, is currently in its infancy, and further work is required to determine the impact of this crosstalk in different endocrine and/or intestinal disease states.

Beyond its capacity as an endocrine organ, the intestinal epithelium forms a barrier between the internal environment and external factors and plays a key role in the orchestration of host defense mechanisms. The involvement of EECs in these processes takes a variety of forms. A key gut hormone that has been implicated is GLP2, which has established effects on mucosal defense and gut barrier function as well as mucosal growth and absorptive capacity (116). EECs sense inflammatory mediators, such as interleukins, and are also capable of detecting bacterial antigens. It is now known that 5-HT-secreting enterochromaffin cells and EEC lines (such as LCC-18) express TLRs (117-119). Stimulation of TLRs in intestinal epithelial cells induces the activation of NF- $\mathrm{BB}$ and the release of inflammatory cytokines and antimicrobial peptides $(120,121)$. 5-HT is known to have emetic properties, and therefore activation of TLRs on enterochromaffin cells could protect against toxic substances. CCK-expressing cell lines were shown to respond to activation with TLR agonists such as bacterial LPS by secreting CCK and stimulating production of inflammatory cytokines, which 
activate innate and adaptive immune responses $(118,119)$. Oral administration of LPS and other TLR agonists elevated serum CCK levels in mice (118). Interestingly, gastrointestinal infection is associated with elevated CCK levels, which is thought to account for the commonly experienced reduction in food intake (122).

\section{Enteroendocrine system and enteric nervous system crosstalk}

Enteric nervous system influence on gut hormone release. Gut hormone secretion is known to be under a certain degree of neurohormonal control, and it has been postulated that these signals may be involved in crosstalk between different regions of the gastrointestinal tract. The activity of EECs is modulated by signals from other EEC populations, as mentioned above, and from the enteric nervous system (ENS), a network of nerve fibers and ganglia innervating the intestine that interacts with branches of both the parasympathetic and sympathetic nervous systems (ref. 123 and Figure 3). Signaling within the ENS involves GPCR activation and, in addition to acetylcholine and noradrenaline, utilizes a large number of peptide neurotransmitters including calcitonin gene-related peptide (CGRP), vasoactive intestinal peptide (VIP), pituitary adenylate cyclase-activating protein (PACAP), galanin, and the bombesin-related neuromedin B (NMB) and gastrinreleasing peptide (GRP).

In isolated perfused porcine ileum, noradrenaline inhibited GLP1 secretion, an effect that was abolished by the non-selective $\alpha$-adrenergic antagonist phentolamine. On the other hand, cholinergic stimulation was found to promote GLP1 secretion (124). In accordance with this, GLP1 release from fetal rat intestinal cultures was stimulated by the muscarinic receptor agonist bethanecol (14) and was inhibited by an M1 receptor antagonist (125). In rats, lipid-induced GLP1 secretion was also inhibited by atropine and M1 muscarinic receptor blockade (125).

Stimulatory pathways. In vitro data obtained using fetal rat intestinal cell cultures revealed that GLP1 secretion was stimulated by CGRP, bombesin, and the bombesin-related GRP (14). CGRP also increased GLP1 release in isolated perfused rat ileum (126). The heterodimeric CGRP receptor was recently shown to be the only neuropeptide receptor enriched in ghrelin cells. In line with its $\mathrm{G}_{\mathrm{s}}$-coupling, CGRP receptor activation dose-dependently stimulated ghrelin secretion from primary gastric mucosal cultures (48).

In humans, GRP has been shown to stimulate the release of multiple gut hormones including CCK and GIP (127). The bombesin-related peptides, NMB and GRP, act via their respective $G_{\mathrm{q}}$ coupled receptors, BB1 and BB2 (128). BB2-deficient mice exhibit impaired GLP1 release in response to a gastric glucose load (129). PACAP has been reported to trigger the release of a number of gut hormones including CCK, GLP1, and PYY $(72,130,131)$. PACAP activates three receptors, PAC1, VPAC1, and VPAC2; VPAC1 and VPAC2 are also activated by VIP (131).

Inhibitory pathways. Galanin is widely expressed in the ENS and, along with somatostatin, is another inhibitor of EEC secretion. In isolated perfused rat ileum, galanin inhibited GIP-stimulated GLP1 release (132); in vitro, galanin inhibited the secretion of several gut hormones including CCK and GLP1 $(133,134)$. These findings are in line with reported effects of galanin in humans, which include a robust inhibition of gastrointestinal motility together with suppression of several gut peptides including PYY and GLP1 but not GIP (135). Galanin is likely to act via a $\mathrm{G}_{\mathrm{i}}$-coupled, pertussis-sensitive galanin receptor (134). Interestingly, recent publications reported significantly elevated plasma galanin levels in patients with obesity and diabetes $(136,137)$.

Cannabinoid receptors are expressed throughout the gastrointestinal tract and are predominantly found in enteric nerve fibers $(138,139)$. Expression of the $\mathrm{G}_{\mathrm{i}}$-coupled cannabinoid receptor 1 (CNR1, also referred to as CB1) is high in I cells (112) and K cells, where it preferentially inhibits GIP over GLP1 release via a reduction in intracellular cAMP (94).

\section{Sensing by the ENS}

Sensing of the luminal milieu, mediated by EECs, is communicated to the ENS via the paracrine action of gut hormones such as CCK and GLP1. Using GLP1 receptor-Cre (Glp1r-Cre) mice crossed with fluorescent reporter strains, GLP1R ${ }^{+}$neuronal cell bodies were recently identified in the ENS (140), confirming earlier immunohistochemistry findings (141). Subsets of enteric neurons are also thought to express CCK1 and GLP2 receptors $(142,143)$ and have additionally been found to contain $R x f p 4$, potentially mediating responses to the orexigenic gut hormone INSL5 (20). Whether these receptors are located in the same or in different individual neurons remains to be established.

Interestingly, enteric neurons appear to be capable of sensing nutrients directly (Figure 3). However, the fact that mucosal ENS innervation does not extend to the gut lumen implies that enteric neurons are predominantly exposed to absorbed nutrients and local metabolic products. FFAR3 has been detected in submucosal and myenteric ganglia (107) and may underlie actions of SCFAs on enteric neuronal activity (144-146). Counterstaining with antibodies against VIP revealed co-localization with FFAR3, indicating that SCFAs may be sensed by secretomotor neurons involved in the regulation of water and electrolyte secretion (107). Enteric neuron nutrient responsiveness has also been demonstrated for glucose, amino acids, and fatty acids (147-149).

GPBAR1 expression was recently demonstrated in inhibitory motor neurons in the myenteric plexus (150), and in light of reports that BAs delay gastric emptying, it led the authors to hypothesize that GPBAR1 may modulate gastrointestinal motility, not only by mediating GLP1 release but also through direct activation of inhibitory enteric neurons.

It is, therefore, becoming increasingly apparent that EECs must be capable of a sophisticated integration of stimulatory and inhibitory signals from multiple sources, which in turn results in the secretion of gut hormones that act not only centrally and via the vagus nerve but also within the ENS. Dissecting how individual components of this complex regulatory network influence gut hormone secretion requires further exploration. Utilization of ex vivo perfused intestine models or other experimental techniques maintaining a polarized epithelium, such as Ussing chambers, will be important.

\section{Concluding remarks}

Gut chemosensing fulfills a fundamental role in postprandial physiology, orchestrating tailored multi-effector responses to ingested nutrients. Significant progress has been made in elucidating the 
molecular mechanisms underlying nutrient-stimulated gut hormone release. However, our understanding of the additional complexity bestowed by neurohormonal fine-tuning of gut hormone secretion and bidirectional communication between the enteroendocrine system and the ENS remains poor. Nevertheless, it is becoming increasingly clear that factors that alter gut motility, and thus alter the delivery of nutrients and exposure of the gut to nutrients, can have profound implications for gut hormone release. It is believed that the markedly elevated levels of GLP1 and PYY following gastric bypass surgery are linked to enhanced delivery of nutrients to the distal intestine and underlie many of the beneficial outcomes on weight loss and glycemia (1). The therapeutic potential of gut hormones has already been established, particularly with regard to GLP1 mimetics, which modulate the incretin axis to improve glucose homeostasis. It is hoped that treatments based on targeting endogenous secretion from EECs will have the added benefit of releasing multiple gut hormones with effects on both glucose control and appetite. A deeper understanding of the complex inner workings of the intestinal neuroendocrine network, and how they collectively regulate postprandial physiology, might further facilitate the exploitation of these systems and the development of novel therapeutic strategies.

\section{Acknowledgments}

Research in the Reimann and Gribble laboratories is currently funded by the Wellcome Trust (grants 084210/Z/07/Z and 088357/Z/09/Z), Full4Health (grants FP7/2011-2015 and 266408), and the Medical Research Council (MRC) (grant MRC_ MC_UU_12012/3).

Address correspondence to: Fiona Gribble or Frank Reimann, Metabolic Research Laboratories and MRC Metabolic Diseases Unit, Level 4, WT-MRC Institute of Metabolic Science, Box 289, Addenbrooke's Hospital, Cambridge CB2 OQQ, United Kingdom. Phone: 44.1223.336746; E-mail: fmg23@cam.ac.uk (F. Gribble). Phone: 44.1223.746796; E-mail: fr222@cam.ac.uk (F. Reimann).
1. Holst J. Enteroendocrine secretion of gut hormones in diabetes, obesity and after bariatric surgery. Curr Opin Pharmacol. 2013;13(6):983-988.

2. Bohórquez D, Chandra R, Samsa L, Vigna S, Liddle R. Characterization of basal pseudopodlike processes in ileal and colonic PYY cells. J Mol Histol. 2011;42(1):3-13.

3. Liou A, et al. The G-protein-coupled receptor GPR40 directly mediates long-chain fatty acidinduced secretion of cholecystokinin. Gastroenterology. 2011;140(3):903-912.

4. Parker H, Habib A, Rogers G, Gribble F, Reimann F. Nutrient-dependent secretion of glucose-dependent insulinotropic polypeptide from primary murine K cells. Diabetologia. 2009;52(2):289-298.

5. Reimann F, et al. Glucose sensing in L cells: a primary cell study. Cell Metab. 2008;8(6):532-539.

6. Habib A, et al. Overlap of endocrine hormone expression in the mouse intestine revealed by transcriptional profiling and flow cytometry. Endocrinology. 2012;153(7):3054-3065.

7. Egerod K, et al. A major lineage of enteroendocrine cells coexpress CCK, secretin, GIP, GLP-1, PYY, and neurotensin but not somatostatin. Endocrinology. 2012;153(12):5782-5795.

8. Engelstoft M, Egerod K, Lund M, Schwartz T. Enteroendocrine cell types revisited. Curr Opin Pharmacol. 2013;13(6):912-921.

9. Sjölund K, Sandén G, Håkanson R, Sundler F. Endocrine cells in human intestine: an immunocytochemical study. Gastroenterology. 1983;85(5):1120-1130.

10. van der Wielen N, et al. Cross-species comparison of genes related to nutrient sensing mechanisms expressed along the intestine. PLoS One. 2014;9(9):e107531.

11. Bado A, et al. The stomach is a source of leptin. Nature. 1998;394(6695):790-793.

12. Cammisotto P, Bendayan M. A review on gastric leptin: the exocrine secretion of a gastric hormone. Anat Cell Biol. 2012;45(1):1-16.

13. Mix H, et al. Expression of leptin and leptin receptor isoforms in the human stomach. Gut.
2000;47(4):481-486.

14. Brubaker P. Regulation of intestinal proglucagonderived peptide secretion by intestinal regulatory peptides. Endocrinology. 1991;128(6):3175-3182.

15. Schubert M. Gastric secretion. Curr Opin Gastroenterol. 2010;26(6):598-603.

16. Date Y, et al. Ghrelin, a novel growth hormonereleasing acylated peptide, is synthesized in a distinct endocrine cell type in the gastrointestinal tracts of rats and humans. Endocrinology. 2000;141(11):4255-4261.

17. Kirchner H, Heppner KM, Tschop MH. The role of ghrelin in the control of energy balance. Handb Exp Pharmacol. 2012;(209):161-184.

18. Verhulst PJ, Depoortere I. Ghrelin's second life: from appetite stimulator to glucose regulator. World J Gastroenterol. 2012;18(25):3183-3195.

19. Rasmussen BA, et al. Jejunal leptin-PI3K signaling lowers glucose production. Cell Metab. 2014;19(1):155-161.

20. Grosse J, et al. Insulin-like peptide 5 is an orexigenic gastrointestinal hormone. Proc Natl Acad Sci U S A . 2014;111(30):11133-11138.

21. Eissele R, et al. Glucagon-like peptide-1 cells in the gastrointestinal tract and pancreas of rat, pig and man. Eur J Clin Invest. 1992;22(4):238-291.

22. Cox HM. Peptide YY: a neuroendocrine neighbor of note. Peptides. 2007;28(2):345-351.

23. Larsson L, Holst J, Håkanson R, Sundler F. Distribution and properties of glucagon immunoreactivity in the digestive tract of various mammals: an immunohistochemical and immunochemical study. Histochemistry. 1975;44(4):281-290.

24. Bohórquez D, et al. An enteroendocrine cellenteric glia connection revealed by 3D electron microscopy. PLoS One. 2014;9(2):e89881.

25 . Tolhurst G, et al. Glutamine triggers and potentiates glucagon-like peptide-1 secretion by raising cytosolic $\mathrm{Ca}^{2+}$ and cAMP. Endocrinology. 2011;152(2):405-413.

26. Chaikomin R, et al. Effects of mid-jejunal compared to duodenal glucose infusion on peptide hormone release and appetite in healthy men. Regul Pept. 2008;150(1):38-42.
27. Hasegawa $\mathrm{H}$, et al. Oral glucose ingestion stimulates cholecystokinin release in normal subjects and patients with non-insulin-dependent diabetes mellitus. Metabolism. 1996;45(2):196-202.

28. Parker BA, Doran S, Wishart J, Horowitz M, Chapman IM. Effects of small intestinal and gastric glucose administration on the suppression of plasma ghrelin concentrations in healthy older men and women. Clin Endocrinol (Oxf). 2005;62(5):539-546.

29. Ritzel U, Fromme A, Ottleben M, Leonhardt U, Ramadori G. Release of glucagon-like peptide-1 (GLP-1) by carbohydrates in the perfused rat ileum. Acta Diabetol. 1997;34(1):18-21.

30. Shima K, Suda T, Nishimoto K, Yoshimoto S. Relationship between molecular structures of sugars and their ability to stimulate the release of glucagon-like peptide-1 from canine ileal loops. Acta Endocrinol (Copenh). 1990;123(4):464-470.

31. Sykes S, Morgan L, English J, Marks V. Evidence for preferential stimulation of gastric inhibitory polypeptide secretion in the rat by actively transported carbohydrates and their analogues. JEndocrinol.1980;85(2):201-207.

32. Gribble F, Williams L, Simpson A, Reimann F. A novel glucose-sensing mechanism contributing to glucagon-like peptide-1 secretion from the GLUTag cell line. Diabetes. 2003;52(5):1147-1154.

33. Parker $\mathrm{H}$, et al. Predominant role of active versus facilitative glucose transport for glucagon-like peptide-1 secretion. Diabetologia. 2012;55(9):2445-2455.

34. Gorboulev V, et al. $\mathrm{Na}(+)$-D-glucose cotransporter SGLT1 is pivotal for intestinal glucose absorption and glucose-dependent incretin secretion. Diabetes. 2012;61(1):187-196.

35. Powell D, et al. LX4211 increases serum glucagonlike peptide 1 and peptide YY levels by reducing sodium/glucose cotransporter 1 (SGLT1)-mediated absorption of intestinal glucose. J Pharmacol Exp Ther. 2013;345(2):250-259.

36. Stephens J, et al. Effects of short-term therapy with glibenclamide and repaglinide on incretin hormones and oxidative damage associated 
with postprandial hyperglycaemia in people with type 2 diabetes mellitus. Diabetes Res Clin Pract. 2011;94(2):199-206.

37. Kuhre R, et al. Fructose stimulates GLP-1 but not GIP secretion in mice, rats, and humans. Am J Physiol Gastrointest Liver Physiol. 2014;306(7):G622-G630.

38. Jang $\mathrm{H}$, et al. Gut-expressed gustducin and taste receptors regulate secretion of glucagon-like peptide-1. Proc Natl Acad Sci U S A. 2007;104(38):15069-15074.

39. Margolskee R, et al. T1R3 and gustducin in gut sense sugars to regulate expression of $\mathrm{Na}^{+}-$ glucose cotransporter 1. Proc Natl Acad Sci U S A. 2007;104(38):15075-15080.

40. Young R, et al. Disordered control of intestinal sweet taste receptor expression and glucose absorption in type 2 diabetes. Diabetes. 2013;62(10):3532-3541.

41. Geraedts MC, et al. Transformation of postingestive glucose responses after deletion of sweet taste receptor subunits or gastric bypass surgery. Am J Physiol Endocrinol Metab. 2012;303(4):E464-E474.

42. Fujita $Y$, et al. Incretin release from gut is acutely enhanced by sugar but not by sweeteners in vivo. Am J Physiol Endocrinol Metab. 2009;296(3):E473-E479.

43. Ma J, et al. Effect of the artificial sweetener, sucralose, on gastric emptying and incretin hormone release in healthy subjects. Am JPhysiol Gastrointest Liver Physiol. 2009;296(4):G735-G739.

44. Batterham R, et al. Critical role for peptide YY in protein-mediated satiation and body-weight regulation. Cell Metab. 2006;4(3):223-233.

45. Reimann F, Williams L, da Silva Xavier G, Rutter G, Gribble F. Glutamine potently stimulates glucagon-like peptide-1 secretion from GLUTag cells. Diabetologia. 2004;47(9):1592-1601.

46. Greenfield J, et al. Oral glutamine increases circulating glucagon-like peptide 1 , glucagon, and insulin concentrations in lean, obese, and type 2 diabetic subjects. Am J Clin Nutr. 2009;89(1):106-113.

47. Mace O, Schindler M, Patel S. The regulation of K- and L-cell activity by GLUT2 and the calciumsensing receptor CasR in rat small intestine. JPhysiol. 2012;590(pt 12):2917-2936.

48. Engelstoft MS, et al. Seven transmembrane G protein-coupled receptor repertoire of gastric ghrelin cells. Mol Metab. 2013;2(4):376-392.

49. Liou A, et al. The extracellular calcium-sensing receptor is required for cholecystokinin secretion in response to L-phenylalanine in acutely isolated intestinal I cells. Am J Physiol Gastrointest Liver Physiol. 2011;300(4):G538-G546.

50. Wang J, et al. Umami receptor activation increases duodenal bicarbonate secretion via glucagonlike peptide-2 release in rats. J Pharmacol Exp Ther. 2011;339(2):464-473.

51. Oya M, et al. The G protein-coupled receptor family C group 6 subtype A (GPRC6A) receptor is involved in amino acid-induced glucagon-like peptide-1 secretion from GLUTag cells. J Biol Chem. 2013;288(7):4513-4521.

52. Cordier-Bussat M, et al. Peptones stimulate both the secretion of the incretin hormone glucagonlike peptide 1 and the transcription of the proglu- cagon gene. Diabetes. 1998;47(7):1038-1045.

53. Reimer R, et al. A human cellular model for studying the regulation of glucagonlike peptide-1 secretion. Endocrinology. 2001;142(10):4522-4528.

54. Liou A, et al. Protein hydrolysate-induced cholecystokinin secretion from enteroendocrine cells is indirectly mediated by the intestinal oligopeptide transporter PepT1. Am J Physiol Gastrointest Liver Physiol. 2011;300(5):G895-G902.

55. Matsumura K, Miki T, Jhomori T, Gonoi T, Seino S. Possible role of PEPT1 in gastrointestinal hormone secretion. Biochem Biophys Res Commun. 2005;336(4):1028-1032.

56. Diakogiannaki E, et al. Oligopeptides stimulate glucagon-like peptide-1 secretion in mice through proton-coupled uptake and the calcium-sensing receptor. Diabetologia. 2013;56(12):2688-2696

57. Choi S, et al. GPR93 activation by protein hydrolysate induces CCK transcription and secretion in STC-1 cells. Am J Physiol Gastrointest Liver Physiol. 2007;292(5):G1366-G1375.

58. Elliott R, et al. Glucagon-like peptide-1 (7-36)amide and glucose-dependent insulinotropic polypeptide secretion in response to nutrient ingestion in man: acute post-prandial and 24-h secretion patterns. JEndocrinol.1993;138(1):159-166.

59. Liddle R, Goldfine I, Rosen M, Taplitz R, Williams J. Cholecystokinin bioactivity in human plasma. Molecular forms, responses to feeding, and relationship to gallbladder contraction. J Clin Invest. 1985;75(4):1144-1152.

60. Spiller R, et al. Further characterisation of the 'ileal brake' reflex in man - effect of ileal infusion of partial digests of fat, protein, and starch on jejunal motility and release of neurotensin, enteroglucagon, and peptide YY. Gut. 1988;29(8):1042-1051.

61. Hirasawa A, et al. Free fatty acids regulate gut incretin glucagon-like peptide- 1 secretion through GPR120. Nat Med. 2005;11(1):90-94.

62. Chu Z, et al. A role for intestinal endocrine cell-expressed g protein-coupled receptor 119 in glycemic control by enhancing glucagonlike Peptide- 1 and glucose-dependent insulinotropic Peptide release. Endocrinology. 2008;149(5):2038-2047.

63. Lauffer L, Iakoubov R, Brubaker P. GPR119 is essential for oleoylethanolamide-induced glucagon-like peptide- 1 secretion from the intestinal enteroendocrine L-cell. Diabetes. 2009;58(5):1058-1066

64. Edfalk S, Steneberg O, Edlund H. Gpr40 is expressed in enteroendocrine cells and mediates free fatty acid stimulation of incretin secretion. Diabetes. 2008;57(9):2280-2287.

65. Hara T, Hirasawa A, Ichimura A, Kimura I, Tsujimoto G. Free fatty acid receptors FFAR1 and GPR120 as novel therapeutic targets for metabolic disorders. JPharm Sci. 2011;100(9):3594-3601.

66. Habib A, Richards P, Rogers G, Reimann F, Gribble F. Co-localisation and secretion of glucagon-like peptide 1 and peptide YY from primary cultured human L cells. Diabetologia. 2013;56(6):1413-1416.

67. Hansen K, et al. 2-Oleoyl glycerol is a GPR119 agonist and signals GLP-1 release in humans. J Clin
Endocrinol Metab. 2011;96(9):E1409-E1417.

68. Cox H, et al. Peptide YY is critical for acylethanolamine receptor Gpr119-induced activation of gastrointestinal mucosal responses. Cell Metab. 2010;11(6):532-542.

69. Overton $\mathrm{H}$, et al. Deorphanization of a $\mathrm{G}$ protein coupled receptor for oleoylethanolamide and its use in the discovery of small-molecule hypophagic agents. Cell Metab. 2006;3(3):167-175.

70. Drucker D, Jin T, Asa S, Young T, Brubaker P. Activation of proglucagon gene transcription by protein kinase-A in a novel mouse enteroendocrine cell line. Mol Endocrinol. 1994;8(12):1646-1655.

71. Islam D, et al. Epac is involved in cAMP-stimulated proglucagon expression and hormone production but not hormone secretion in pancreatic alpha- and intestinal L-cell lines. Am JPhysiol Endocrinol Metab. 2009;296(1):E174-E181.

72. Simpson A, et al. Cyclic AMP triggers glucagonlike peptide- 1 secretion from the GLUTag enteroendocrine cell line. Diabetologia. 2007;50(10):2181-2189.

73. Katz L, et al. Effects of JNJ-38431055, a novel GPR119 receptor agonist, in randomized, double-blind, placebo-controlled studies in subjects with type 2 diabetes. Diabetes Obes Metab. 2012;14(8):709-716.

74. Poreba M, et al. Role of fatty acid transport protein 4 in oleic acid-induced glucagon-like peptide-1 secretion from murine intestinal L cells. Am J Physiol Endocrinol Metab. 2012;303(7):E899-E907.

75. Iakoubov R, Ahmed A, Lauffer L, Bazinet R, Brubaker P. Essential role for protein kinase $\mathrm{C} \zeta$ in oleic acid-induced glucagon-like peptide-1 secretion in vivo in the rat. Endocrinology. 2011;152(4):1244-1252.

76. Fatma S, Yakubov R, Anwar K, Hussain M. Pluronic L81 enhances triacylglycerol accumulation in the cytosol and inhibits chylomicron secretion. J Lipid Res. 2006;47(11):2422-2432.

77. Shimotoyodome A, et al. Coingestion of acylglycerols differentially affects glucose-induced insulin secretion via glucose-dependent insulinotropic polypeptide in C57BL/6J mice. Endocrinology. 2009;150(5):2118-2126.

78. Lu W, et al. Chylomicron formation and secretion is required for lipid-stimulated release of incretins GLP-1 and GIP. Lipids. 2012;47(6):571-580.

79. Chandra R, et al. Immunoglobulin-like domain containing receptor 1 mediates fat-stimulated cholecystokinin secretion. JClin Invest. 2013;123(8):3343-3352.

80. Okawa M, et al. Role of MGAT2 and DGAT1 in the release of gut peptides after triglyceride ingestion. Biochem Biophys Res Commun 2009;390(3):377-381.

81. Hata T, et al. JTT-130, a novel intestine-specific inhibitor of microsomal triglyceride transfer protein, suppresses food intake and gastric emptying with the elevation of plasma peptide YY and glucagon-like peptide-1 in a dietary fat-dependent manner. JPharmacol Exp Ther. 2011;336(3):850-856.

82. Flock G, Cao X, Maziarz M, Drucker D. Activation of enteroendocrine membrane progesterone receptors promotes incretin secretion and improves glucose tolerance in mice. Diabetes. 2013;62(1):283-290. 
83. Franckhauser S, et al. Overexpression of Il6 leads to hyperinsulinaemia, liver inflammation and reduced body weight in mice. Diabetologia. 2008;51(7):1306-1316.

84. Holmes A, et al. Prolonged interleukin- 6 administration enhances glucose tolerance and increases skeletal muscle PPAR $\alpha$ and UCP2 expression in rats. J Endocrinol. 2008;198(2):367-374.

85. Adam T, Westerterp-Plantenga M. Activityinduced GLP-1 release in lean and obese subjects. Physiol Behav. 2004;83(3):459-466.

86. Martins C, Morgan L, Bloom S, Robertson M. Effects of exercise on gut peptides, energy intake and appetite. J Endocrinol. 2007;193(2):251-258.

87. Ellingsgaard $\mathrm{H}$, et al. Interleukin- 6 enhances insulin secretion by increasing glucagon-like peptide- 1 secretion from $L$ cells and alpha cells. Nat Med. 2011;17(11):1481-1489.

88. Adrian T, et al. Rectal taurocholate increases L cell and insulin secretion, and decreases blood glucose and food intake in obese type 2 diabetic volunteers. Diabetologia. 2012;55(9):2343-2347.

89. Wu T, et al. Effects of taurocholic acid on glycemic, glucagon-like peptide-1, and insulin responses to small intestinal glucose infusion in healthy humans. JClin Endocrinol Metab. 2013;98(4):E718-E722.

90. Patti M, et al. Serum bile acids are higher in humans with prior gastric bypass: potential contribution to improved glucose and lipid metabolism. Obesity (Silver Spring). 2009;17(9):1671-1677.

91. Pournaras D, et al. The role of bile after Rouxen-Y gastric bypass in promoting weight loss and improving glycaemic control. Endocrinology. 2012;153(8):3613-3619.

92. Parker H, et al. Molecular mechanisms underlying bile acid-stimulated glucagon-like peptide-1 secretion. Br J Pharmacol. 2012;165(2):414-423.

93. Thomas C, et al. TGR5-mediated bile acid sensing controls glucose homeostasis. Cell Metab. 2009;10(3):167-177.

94. Moss C, et al. Somatostatin receptor 5 and cannabinoid receptor 1 activation inhibit secretion of glucose-dependent insulinotropic polypeptide from intestinal K cells in rodents. Diabetologia. 2012;55(11):3094-3103.

95. Batterham RL, et al. Inhibition of food intake in obese subjects by peptide YY3-36. $N$ Engl J Med. 2003;349(10):941-948.

96. Degen L, et al. Effect of CCK-1 receptor blockade on ghrelin and PYY secretion in men. Am J Physiol Regul Integr Comp Physiol. 2007;292(4):R1391-R1399.

97. Rudovich NN, et al. Metabolomic linkage reveals functional interaction between glucosedependent insulinotropic polypeptide and ghrelin in humans. Am J Physiol Endocrinol Metab. 2011;301(4):E608-E617.

98. Backhed F, et al. The gut microbiota as an environmental factor that regulates fat storage. Proc Natl Acad Sci U S A. 2004;101(44):15718-15723.

99. Ley RE, Turnbaugh PJ, Klein S, Gordon JI. Microbial ecology: human gut microbes associated with obesity. Nature. 2006;444(7122):1022-1023.

100.Ridaura VK, et al. Gut microbiota from twins discordant for obesity modulate metabolism in mice. Science. 2013;341(6150):1241214.

101. Turnbaugh PJ, et al. An obesity-associated gut microbiome with increased capacity for energy harvest. Nature. 2006;444(7122):1027-1031.

102. Brown A, et al. The Orphan $G$ protein-coupled receptors GPR41 and GPR43 are activated by propionate and other short chain carboxylic acids. J Biol Chem. 2003;278(13):11312-11319.

103. Le Poul E, et al. Functional characterization of human receptors for short chain fatty acids and their role in polymorphonuclear cell activation. J Biol Chem. 2003;278(28):25481-25489.

104.Karaki S, et al. Short-chain fatty acid receptor, GPR43, is expressed by enteroendocrine cells and mucosal mast cells in rat intestine. Cell Tissue Res. 2006;324(3):353-360.

105. Karaki S, et al. Expression of the short-chain fatty acid receptor, GPR43, in the human colon. JMol Histol. 2008;39(2):135-142.

106. Tazoe H, et al. Expression of short-chain fatty acid receptor GPR41 in the human colon. Biomed Res. 2009;30(3):149-156.

107. Nøhr M, et al. GPR41/FFAR3 and GPR43/FFAR2 as cosensors for short-chain fatty acids in enteroendocrine cells vs FFAR3 in enteric neurons and FFAR2 in enteric leukocytes. Endocrinology. 2013;154(10):3552-3564.

108. Tolhurst G, et al. Short-chain fatty acids stimulate glucagon-like peptide-1 secretion via the G-protein-coupled receptor FFAR2. Diabetes. 2012;61(2):364-371.

109. Seljeset S, Siehler S. Receptor-specific regulation of ERK1/2 activation by members of the "free fatty acid receptor" family. J Recept Signal Transduct Res. 2012;32(4):196-201.

110. Lim G, et al. Insulin regulates glucagon-like peptide- 1 secretion from the enteroendocrine $\mathrm{L}$ cell. Endocrinology. 2009;150(2):580-591.

111. Psichas A, et al. The short chain fatty acid propionate stimulates GLP-1 PYY secretion via free fatty acid receptor 2 in rodents [published online ahead of print August 11, 2014]. Int JObes (Lond). doi:10.1038/ijo.2014.153.

112. Sykaras A, Demenis C, Case R, McLaughlin J, Smith C. Duodenal enteroendocrine I-cells contain mRNA transcripts encoding key endocannabinoid and fatty acid receptors. PLoS One. 2012;7(8):e42373

113. Clarke G, et al. Minireview: Gut microbiota: the neglected endocrine organ. Mol Endocrinol. 2014;28(8):1221-1238.

114. Nicholson JK, et al. Host-gut microbiota metabolic interactions. Science. 2012;336(6086):1262-1267.

115. Chimerel C, Emery E, Summers DK, Keyser U, Gribble FM, Reimann F. Bacterial metabolite indole modulates incretin secretion from intestinal enteroendocrine L-cells. Cell Rep. 2014;9(4):1202-1208.

116. Drucker DJ, Yusta B. Physiology and pharmacology of the enteroendocrine hormone glucagonlike peptide-2. Annu Rev Physiol. 2014;76:561-583.

117. Bogunovic M, et al. Enteroendocrine cells express functional Toll-like receptors. Am J Physiol Gastrointest Liver Physiol. 2007;292(6):G1770-G1783.

118. Palazzo M, et al. Activation of enteroendocrine cells via TLRs induces hormone, chemokine, and defensin secretion. Jimmunol. 2007;178(7):4296-4303.

119. Selleri S, et al. Induction of pro-inflammatory programs in enteroendocrine cells by the Toll- like receptor agonists flagellin and bacterial LPS. Int Immunol. 2008;20(8):961-970.

120. Akhtar M, Watson JL, Nazli A, McKay DM. Bacterial DNA evokes epithelial IL-8 production by a MAPK-dependent, NF- $\kappa \mathrm{B}$-independent pathway. FASEB J. 2003;17(10):1319-1321.

121. Suzuki M, Hisamatsu T, Podolsky DK. $\gamma$-Interferon augments the intracellular pathway for lipopolysaccharide (LPS) recognition in human intestinal epithelial cells through coordinated up-regulation of LPS uptake and expression of the intracellular Toll-like receptor 4-MD2 complex. Infect Immun. 2003;71(6):3503-3511.

122. McDermott JR, et al. Immune control of food intake: enteroendocrine cells are regulated by $\mathrm{CD}^{+}{ }^{+} \mathrm{T}$ lymphocytes during small intestinal inflammation. Gut. 2006;55(4):492-497.

123. Reimann F, Tolhurst G, Gribble F. G-proteincoupled receptors in intestinal chemosensation. Cell Metab. 2012;15(4):421-431.

124. Hansen L, Lampert S, Mineo H, Holst J. Neural regulation of glucagon-like peptide-1 secretion in pigs. Am J Physiol Endocrinol Metab. 2004;287(5):E939-E947.

125. Anini Y, Hansotia T, Brubaker P. Muscarinic receptors control postprandial release of glucagon-like peptide-1: in vivo and in vitro studies in rats. Endocrinology. 2002;143(6):2420-2426.

126. Herrmann-Rinke C, McGregor G, Göke B. Calcitonin gene-related peptide potently stimulates glucagon-like peptide-1 release in the isolated perfused rat ileum. Peptides. 2000;21(3):431-437.

127. Ghatei M, et al. Bombesin: action on gut hormones and calcium in man. JClin Endocrinol Metab. 1982;54(5):980-985.

128. Jensen R, Battey J, Spindel E, Benya R. International Union of Pharmacology. LXVIII. Mammalian bombesin receptors: nomenclature, distribution, pharmacology, signaling, and functions in normal and disease states. Pharmacol Rev. 2008;60(1):1-42.

129. Persson K, et al. Reduced GLP-1 and insulin responses and glucose intolerance after gastric glucose in GRP receptor-deleted mice. Am JPhysiol Endocrinol Metab. 2000;279(5):E956-E962.

130.Chang C, Chey W, Braggins L, Coy D, Chang T. Pituitary adenylate cyclase-activating polypeptide stimulates cholecystokinin secretion in STC-1 cells. Am J Physiol. 1996;271(3):G516-G523.

131. Vaudry D, et al. Pituitary adenylate cyclaseactivating polypeptide and its receptors: from structure to functions. Pharmacol Rev. 2000;52(2):269-324.

132. Herrmann-Rinke C, Hörsch D, McGregor G, Göke B. Galanin is a potent inhibitor of glucagonlike peptide-1 secretion from rat ileum. Peptides. 1996;17(4):571-576

133. Chang C, Chey W, Coy D, Chang T. Galanin inhibits cholecystokinin secretion in STC-1 cells. Biochem Biophys Res Commun. 1995;216(1):20-25.

134. Saïfia S, et al. Galanin inhibits glucagon-like peptide-1 secretion through pertussis toxinsensitive $G$ protein and ATP-dependent potas sium channels in rat ileal L-cells. J Endocrinol. 1998;157(1):33-41.

135. Bauer F, et al. Inhibitory effect of galanin on postprandial gastrointestinal motility and gut hormone release in humans. Gastroenterology. 
1989;97(2):260-264.

136. Fang P, Bo P, Shi M, Yu M, Zhang Z. Circulating galanin levels are increased in patients with gestational diabetes mellitus. Clin Biochem. 2013;46(9):831-833.

137. Legakis I, Mantzouridis T, Mountokalakis T. Positive correlation of galanin with glucose in type 2 diabetes. Diabetes Care. 2005;28(3):759-760.

138. Coutts A, Irving A, Mackie K, Pertwee R, Anavi-Goffer S. Localisation of cannabinoid $\mathrm{CB}(1)$ receptor immunoreactivity in the guinea pig and rat myenteric plexus. J Comp Neurol. 2002;448(4):410-422.

139. López-Redondo F, Lees G, Pertwee R. Effects of cannabinoid receptor ligands on electrophysiological properties of myenteric neurones of the guineapig ileum. Br JPharmacol. 1997;122(2):330-334.

140. Richards P, et al. Identification and characterization of GLP-1 receptor-expressing cells using a new transgenic mouse model. Diabetes. 2014;63(4):1224-1233.
141. Amato A, et al. Peripheral motor action of glucagon-like peptide-1 through enteric neuronal receptors. Neurogastroenterol Motil. 2010;22(6):664-e203.

142.de Heuvel E, Wallace L, Sharkey K, Sigalet D. Glucagon-like peptide 2 induces vasoactive intestinal polypeptide expression in enteric neurons via phophatidylinositol 3-kinase- $\gamma$ signaling. Am J Physiol Endocrinol Metab. 2012;303(8):E994-E1005.

143. Patterson L, Zheng H, Berthoud H. Vagal afferents innervating the gastrointestinal tract and CCKA-receptor immunoreactivity. Anat Rec. 2002;266(1):10-20.

144.Bertrand P, Kunze W, Bornstein J, Furness J, Smith M. Analysis of the responses of myenteric neurons in the small intestine to chemical stimulation of the mucosa. Am JPhysiol. 1997;273(2):G422-G435.

145. Neunlist M, Dobreva G, Schemann M. Characteristics of mucosally projecting myenteric neu- rones in the guinea-pig proximal colon. J Physiol. 1999;517(pt 2):533-546.

146.Soret R, et al. Short-chain fatty acids regulate the enteric neurons and control gastrointestinal motility in rats. Gastroenterology. 2010;138(5):1772-1782.

147. Furness J, Rivera L, Cho H, Bravo D, Callaghan B. The gut as a sensory organ. Nat Rev Gastroenterol Hepatol. 2013;10(12):729-740.

148.Liu M, Seino S, Kirchgessner A. Identification and characterization of glucoresponsive neurons in the enteric nervous system. J Neurosci. 1999;19(23):10305-10317.

149. Neunlist M, Schemann M. Nutrient-induced changes in the phenotype and function of the enteric nervous system. J Physiol. 2014; 592(pt 14):2959-2965.

150. Poole D, et al. Expression and function of the bile acid receptor GPBAR1 (TGR5) in the murine enteric nervous system. Neurogastroenterol Motil. 2010;22(7):814-825. 